\section{Clinical features of drug-induced Parkinsonism}

\author{
Nobuko Shiraiwa, ${ }^{1}$ Akira Tamaoka, ${ }^{2}$ \\ Norio Ohkoshi ${ }^{1}$
}

1Course of Neurology, Department of Health Sciences, Tsukuba University of Technology; 2Deparment of Neurology, Graduate School of Comprehensive Human Science, University of Tsukuba, Japan

\begin{abstract}
Drug-induced Parkinsonism is often reversible after withdrawal of the causative drug. Its clinical course, however, is not well understood, as the majority of cases are caused by drugs prescribed by departments outside of neurology. We reviewed 21 cases of drug-induced parkinsonism for several factors, including age, sex, causative drug and reason for prescription, department by which it was prescribed, and outcome. The age at onset ranged from 40 to 87 years, with an average Hoehn and Yahr Scale score of 4, indicating severe disability. Sulpiride was the most commonly observed causative drug (71.4\%). All causative drugs were prescribed in non-neurological departments and over one half were prescribed in non-psychiatric departments; most were prescribed to treat depression or abdominal discomfort. Ten patients $(48 \%)$ were previously diagnosed with a neuromuscular disease, including cerebrovascular diseases and Parkinson's disease. Recovery was observed in 15 cases $(71 \%)$ after withdrawal of the causative drug, but lingering symptoms were observed in the remaining cases. It is suggested that physicians should be more cautious of Parkinsonian side effects when prescribing such drugs.
\end{abstract}

\section{Introduction}

Drug-induced Parkinsonism (DIP) is the second most prevalent cause of secondary Parkinsonism. Its symptoms, which include tremor, rigidity, bradykinesia, and gait disturbance, are very similar to those of Parkinson's disease (PD). Initially reported as a complication of antipsychotics, it was later recognized as a common complication of antidepressants, calcium channel antagonists, gastrointestinal prokinetics, antiepileptic drugs, and many other compounds. ${ }^{1,2}$ DIP is particularly burdensome for the elderly and its management includes the recognition of symptoms and identification of risk factors and offending agents. Prompt discontinuation of the causative agent often leads to marked improvement, though the condition might persist or remit slowly in up to $10 \%$ of patients. These patients are often suspected of concomitantly developing PD. ${ }^{3}$ DIP shows more rapid progress, symmetry of symptoms, relative absence of rest tremor, and coexistence of oro-mandibular dyskinesias compared with PD. However, differentiating DIP from PD in such cases is difficult.2,4

Additionally, DIP is frequently overlooked ${ }^{5-7}$ and its clinical course is not well understood because the majority of cases are caused by drugs prescribed by departments outside of neurology. Therefore, we aimed to examine the clinical course of DIP.

\section{Materials and Methods}

We reviewed 21 cases of drug-induced Parkinsonism and gathered information on 13 different parameters to study the clinical course of the illness. These parameters included: age at onset, sex, whether the case was inpatient or outpatient, maximum Hoehn and Yahr Scale score, causative drug, reason for prescribing the drug, department by which the drug was prescribed, description of any involuntary movement, brain abnormalities (as determined by magnetic resonance imaging [MRI]), use of any anti-parkinsonism drugs, neuromuscular diseases before DIP onset, outcome, duration of causative drug use before DIP onset, time to recovery after withdrawal of causative drug and difficulties after drug withdrawal (Table 1).

\section{Results}

The age at onset ranged from 40 to 87 years, with $90 \%$ of patients over the age of 65 years and a male: female ratio of 2:5. The average Hoehn and Yahr Scale score was 4 , which was indicative of severe disability. Two patients showed oromandibular dyskinesia (Table 1). Sulpiride was the most common causative drug $(71.4 \%)$; other drugs included tiapride, metoclopramide, maprotiline, haloperidol, and risperidone (Figure 1). All causative drugs were prescribed in departments that did not specialize in neurology, with a large portion prescribed by psychiatric departments (eight cases; 38.1\%). In the remaining 13 cases $(61.9 \%)$, drugs were prescribed in a nonpsychiatric department, which included seven by a general practitioner, three in a
Correspondence: Nobuko Shiraiwa, Course of Neurology, Department of Health Sciences, Tsukuba University of Technology, Japan. Tel./Fax: +81298589538 .

E-mail: shiraiwa@xa2.so-net.ne.jp

Key words: Drug-induced Parkinsonism sulpiride; Parkinson's disease; malignant syndrome; oromandibular dyskinesia.

Acknowledgments: we would like to thank Editage (www.editage.jp) for English language editing.

Conflicts of interests: the authors declare no potential conflicts of interest.

Received for publication: 19 September 2018 Revision received: 10 October 2018.

Accepted for publication: 8 November 2018.

This work is licensed under a Creative Commons Attribution NonCommercial 4.0 License (CC BY-NC 4.0).

(C) Copyright N. Shiraiwa et al., 2018

Licensee PAGEPress, Italy

Neurology International 2018; 10:7877

doi:10.4081/ni.2018.7877

general hospital's surgical department, two in a general hospital's internal medicine department, and one in a nursing home (Figure 2A). Commonly cited reasons for prescription included depression (29\%), other psychiatric symptoms (33\%), abdominal discomfort (19\%), and unknown (19\%) (Figure 2B).

Based on clinical symptoms and neuroimaging (MRI and computed tomography) findings, neuromuscular diseases were not present in 11 of the cases $(52 \%)$ before symptom onset. However, these diseases were present in the remaining $48 \%$, which included cerebrovascular diseases (28\%; five vascular dementia, one higher-order dysfunction due to multiple cerebral infarction, and one hemiplegia), three with neurodegenerative diseases (14.3\%; Parkinson's disease, familial spastic paraplegia, and geriatric dementia), and one with depression (4.8\%) (Figure 3A). After withdrawal of the causative medication, 15 of the patients $(71 \%)$ were once again able to walk at home, but three were wheelchairbound and one died from malignant syndrome (Figure 3B).

In 13 patients from 15 patients who were again able to walk, we could examine the relationship between the length of time from first administration of the causative drug to the onset of symptoms and the length of time until recovery after drug withdrawal. It was found that the length of time from first administration of the drug 


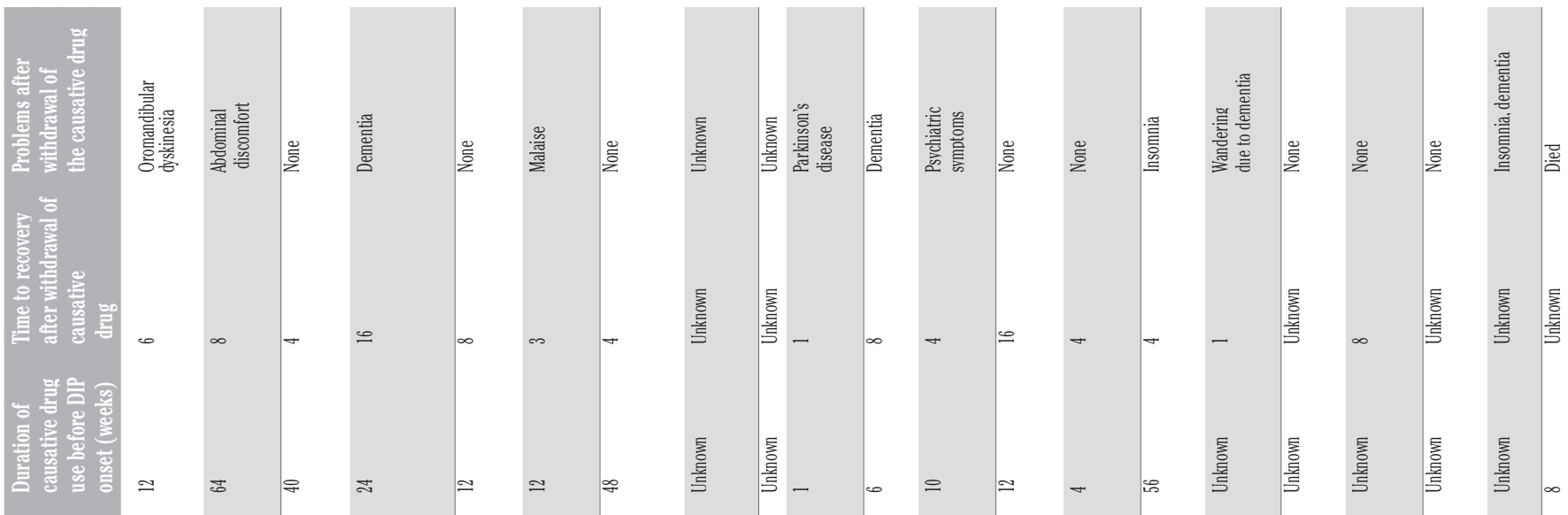

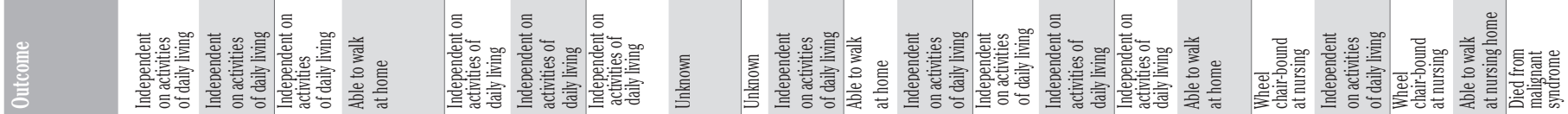

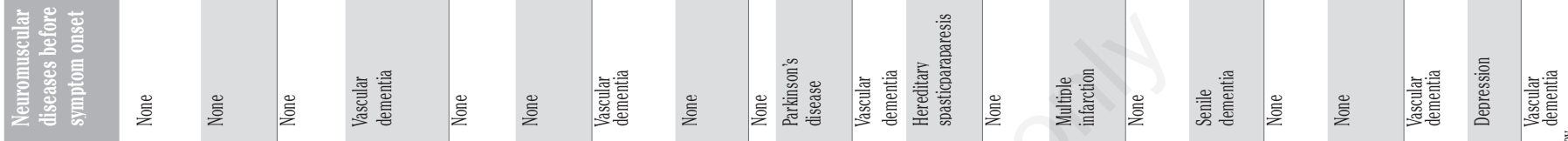

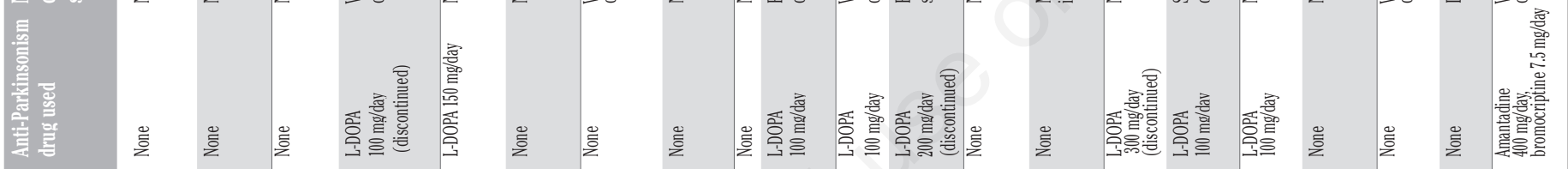

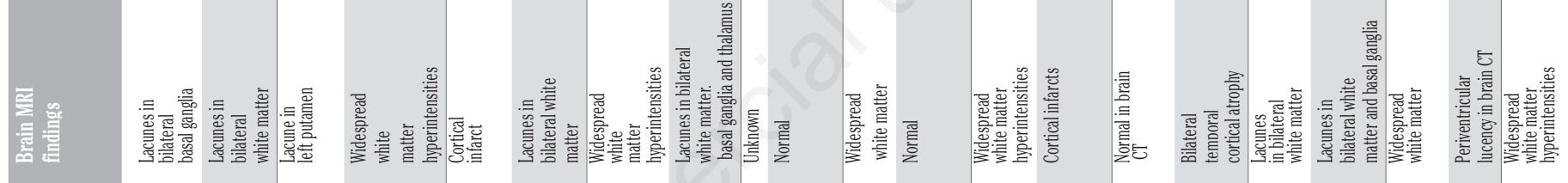

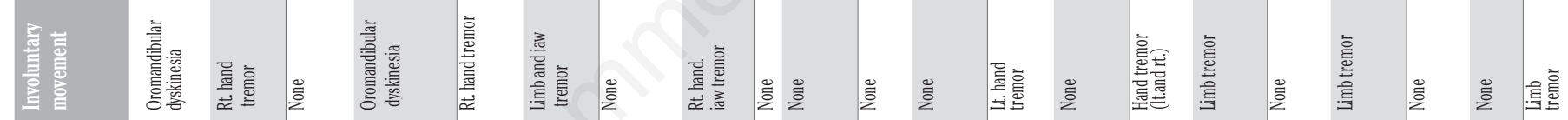

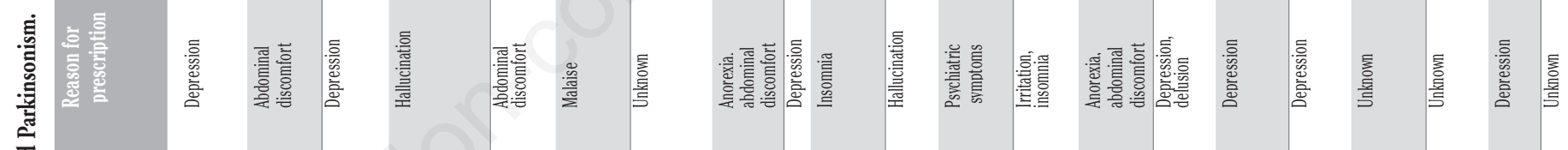

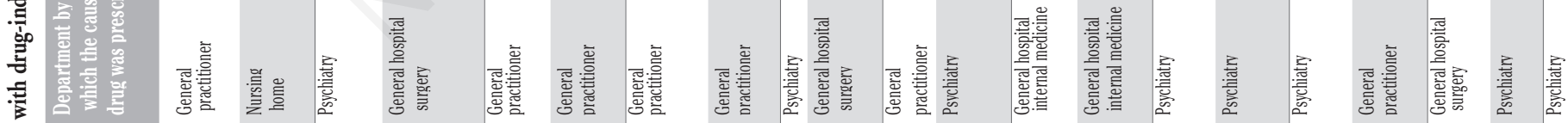

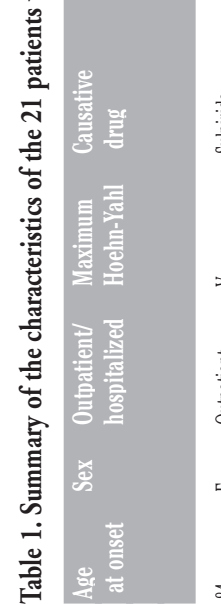


until symptom onset ranged from 1 to 64 weeks (mean 23.2 \pm 42.7 weeks), while the period for recovery ranged from 1 to 16 weeks (average $13.2 \pm 4.7$ weeks). There was no significant correlation between symptom onset and recovery (Figure 4).

\section{Discussion}

Examination of 21 cases of druginduced Parkinsonism (DIP) from our department showed that DIP was more common in elderly women, which is consistent with the known risk factors for DIP. ${ }^{3}$ Additionally, DIP progressed more rapidly than Parkinson's disease. Despite the patients having Hoehn and Yahr Scale ratings between 4 and 5, cessation of the medication resulted in relatively rapid recovery. The patients were able to fully recover and return to their baseline state; 15 of 21 cases $(71.4 \%)$ had good outcomes in that they were able to walk at home after drug withdrawal. However, there were cases in which the patient entered a geriatric facility and became wheelchair-bound, as well as one case of death due to malignant syndrome.

These data suggest that prescription by departments outside of psychiatry accounts for more than $60 \%$ of DIP cases. It is possible that these medications are being prescribed by physicians with a minimal understanding of their dangerous side effects; therefore, more attention from the prescribing physician is required. Lopes-Sendon et $a l .{ }^{3}$ found that the risk factors for developing DIP included older age; female sex; cognitive impairment; potency, dose, and length of treatment; and pre-existing extrapyramidal signs. More attention should be paid to the risk factors of DIP.

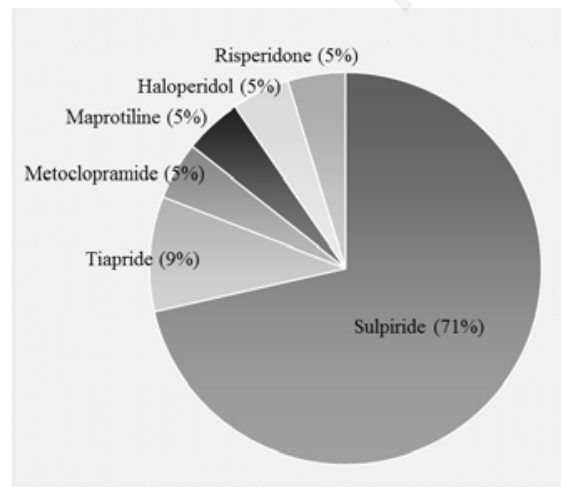

Figure 1. Sulpiride was the most common causative drug (71.4\%). The other causative drugs were tiapride, metoclopramide, maprotiline, haloperidol, and risperidone.

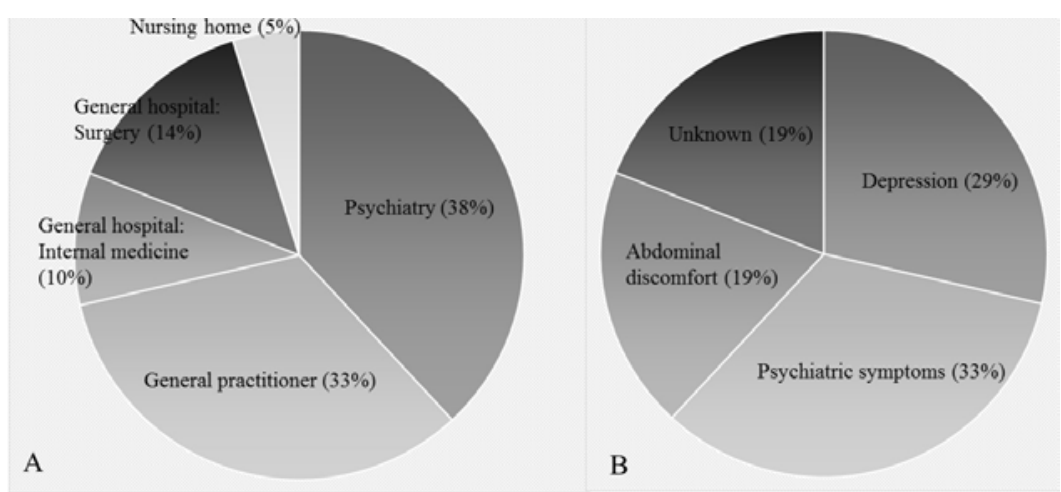

Figure 2. All causative drugs were prescribed in non-neurological departments. Drugs were prescribed in 8 cases $(38.1 \%)$ in psychiatric and in 13 cases $(61.9 \%)$ in non-psychiatric departments, which included prescriptions from seven general practitioners, three general hospital surgeons, two general hospital internal medicine physicians, and one nursing home physician. In total, over half of the causative drugs were prescribed in non-psychiatric departments (A). The main reasons for prescribing were listed as depression (29\%), psychiatric symptoms (33\%), abdominal discomfort (19\%), and unknown (19\%) (B).
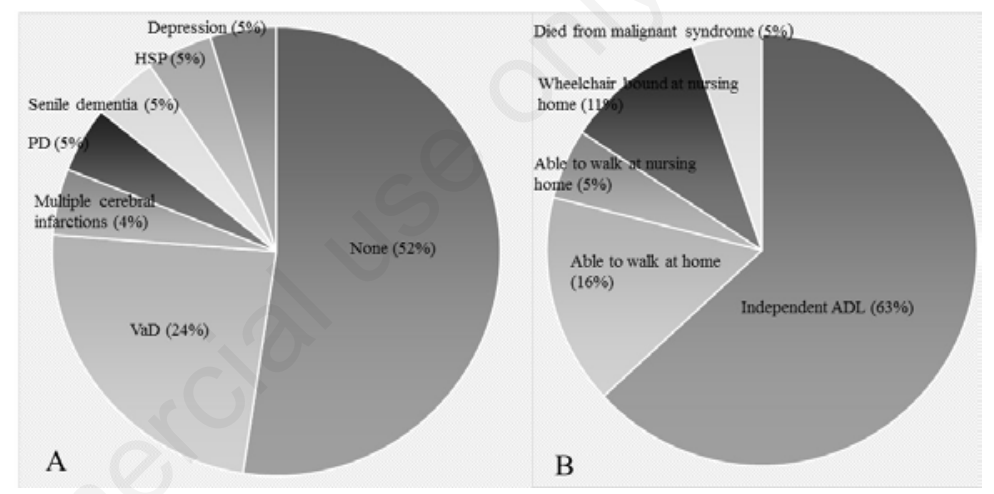

Figure 3. Based on clinical symptoms and neuroimaging findings, 11 of the 21 cases $(52 \%)$ had no presence of neuromuscular diseases before DIP onset. The remaining $48 \%$ did, which included cerebrovascular diseases $(\mathbf{2 8} \%$; five vascular dementia, one higher-order dysfunction due to multiple cerebral infarction, and one hemiplegia), neurodegenerative diseases $(14.3 \%$; one Parkinson's disease, one case of familial spastic paraplegia, and one case of geriatric dementia), and depression (4.8\%) (A). Fifteen of the patients (71\%) had a good outcome in that they were able to walk at home after withdrawal of the causative drug, but three were wheelchair-bound and one died from malignant syndrome (B).

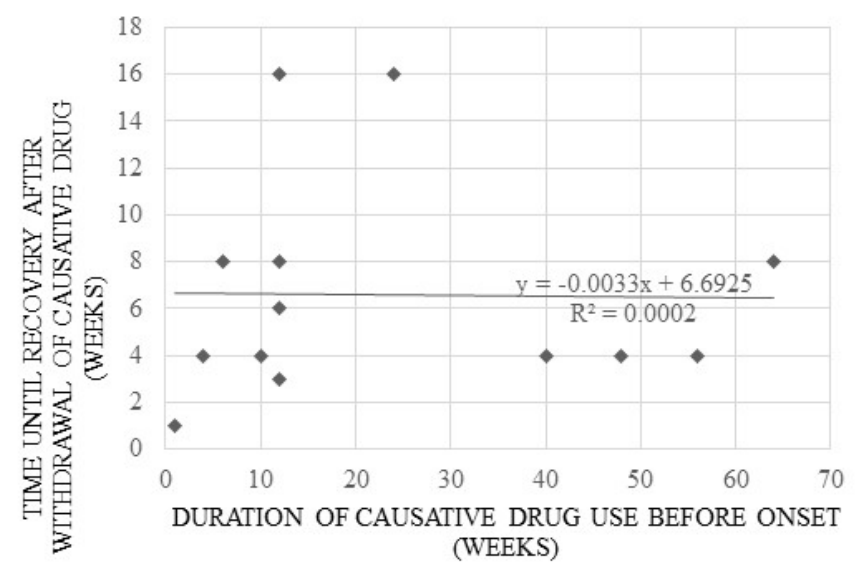

Figure 4. There was no significant correlation between duration of use of the causative drug before DIP onset and time to recovery after drug withdrawal. 
We also found there to be some difficulties associated with drug withdrawal because some cases experienced side effects after withdrawal of the causative drug. For example, some patients experienced abdominal discomfort, psychiatric symptoms, insomnia, or behavioral and psychological symptoms of dementia following withdrawal. In these cases, the symptoms experienced following withdrawal were the main reasons for the prescription. If the medication cannot be withdrawn, the dose should be lowered or the medication switched to reduce the risk of DIP. Additionally, another problem experienced following withdrawal of the causative drug is oromandibular dyskinesia. Shin et al. found that levosulpiride-induced movement disorders are often severe, and are irreversible even after withdrawal of the drug. ${ }^{8}$ In our study, there was a patient who still showed oromandibular dyskinesia after drug withdrawal. Physicians should be also cautious of such symptoms.

\section{Conclusions}

In our study, all of the causative drugs were prescribed in non-neurological departments, and more than $60 \%$ were prescribed in non-psychiatric departments. Approximately $70 \%$ of patients recovered after drug withdrawal; however, the remaining patients did not. We suggest that neurologists pay closer attention to the Parkinsonian side effects of these commonly prescribed drugs.

\section{References}

1. Lopez-Sendon J, Mena MA, de Yebenes JG. Drug-induced Parkinsonism. Expert Opin Drug Saf 2013;12:487-96.

2. Brigo F, Erro R, Marangi A, et al. Differentiating drug-induced parkinsonism from Parkinson's disease: an update on non-motor symptoms and investiga- tions. Parkinsonism Relat Disord 2014;20:808-14.

3. Lopez-Sendon JL, Mena MA, de Yebenes JG. Drug-induced Parkinsonism in the elderly: incidence, management and prevention. Drugs Aging 2012;29:105-18.

4. Hong JY, Sunwoo MK, Oh JS, et al. Persistent drug-induced Parkinsonism in patients with normal dopamine transporter imaging. PLoS One 2016;13:11.

5. Friedman JH. Viewpoint: Challenges in our understanding of neuroleptic induced Parkinsonism. Parkinsonism Relat Disord 2014;20:1325-8.

6. Kuzuhara S. Iatrogenic neurological disorders in old people: A review. Jpn J Geriat 2005;42:21-4.

7. Kuzuhara S. Iatrogenic diseases in the elderly. Jpn J Geriat 1991;28:493-8.

8. Shin HW, Kim MJ, Kim JS, et al. Levosulpiride-induced movement disorders. Mov Disord 2009;24:2249-53. 